\title{
In vivo quantification of ultrasound targeted microbubbles to enhance cancer assessment
}

\author{
Claudia Sciallero ${ }^{a *}$, Emanuele Daglio ${ }^{b}$ and Andrea Trucco ${ }^{a, c}$
}

\begin{abstract}
Contrast-enhanced ultrasound with targeted microbubble contrast agents is an emerging technique for imaging biological processes at the molecular level. The accumulation of targeted microbubbles at tissue sites overexpressing specific molecular markers increases the backscattered signal for noninvasive evaluations of diseases. The aim of this preliminary study was to combine molecular imaging with an in vivo contrast agent quantification to support the early diagnosis of the pathology and to enhance the assessment of neoplastic tissues. Tumor growth was induced by subcutaneous injection of prostate cancer cells in four rats. Microbubbles targeted to tissue factor (TF) were administered. A vascularized region located in proximity to the tumor and centered around the focus depth was analyzed in each animal. The backscattered signals (i.e. the radio-frequency data) were acquired during two different perfusion conditions to evaluate the contribution of attached microbubbles. After image generation by means of a multi-pulse contrast-enhanced technique, a nonlinear regression method based on the support vector machine was employed to estimate the contrast agent concentrations in cubic voxels (1-mm side length). The number of attached microbubbles per $\mathrm{mm}^{3}$ was estimated based on a multi-dimensional vector of features extracted from the processed radio-frequency signals. A significant correlation $(p<0.05)$ between the size of the tumors and the estimated microbubble concentration was found, thus opening the possibility for combining molecular imaging and contrast agent concentration mapping to refine pathology evaluation. Copyright $\odot 2016$ John Wiley \& Sons, Ltd.
\end{abstract}

Keywords: ultrasound medical imaging; molecular imaging; targeted ultrasound contrast agents; quantitative medical ultrasound; nonlinear regression

\section{INTRODUCTION}

Molecular imaging with ultrasound targeted contrast agents (CAs) is based on the same general concept used for the targeting of optical imaging dyes, radiopharmaceuticals and magnetic resonance imaging (MRI) CAs. Specific ligands able to adhere to targets on the cell surface under disease-specific conditions are coupled to the CA particle (1). In the case of ultrasound molecular imaging, the protective shells of gas-filled microbubbles (MBs) comprising ultrasound CAs are typically modified to allow selective binding to cellular epitopes or other receptors (i.e. active targeting). Due to the typical size $(1-8 \mu \mathrm{m})$ of MBs (2), they are restricted to the vasculature, implying that only vascular disease markers can be addressed. Molecular signatures that are overexpressed in regions of angiogenesis or inflammation can be used to localize the CAs due to complementary receptor ligands (3). The resultant accumulation of CAs in the target area increases the backscattered signal. As a consequence, ultrasound imaging with targeted CAs can provide higher sensitivity and specificity with respect to non-targeted agents.

In recent years, a variety of targeted MBs have been prepared and applied to experiments of in vitro cell-specific targeting and in vivo molecular imaging. By comparing non-targeted and targeted MBs, targeted MBs have demonstrated an enhanced acoustic intensity for facilitating a noninvasive evaluation of early angiogenesis for real-time imaging (4-7). Angiogenesis plays a critical role in many disease processes, such as tumor growth and metastasis. An effective quantification of the accumulated MBs at the target sites provides additional information for more accurate cancer assessments. This parameter is particularly attractive supposing that $\mathrm{MB}$ retention is primarily influenced by the ligand density. In the case of non-targeted MBs, ultrasound images are supplemented by the quantification of tissue perfusion, that is the quantification of blood flow using the image intensity of a region of interest (ROI) as a function of time, to differentiate malignant from benign tumors in the liver and to enhance the ultrasound sensitivity for diagnosing breast and prostate tumors $(4,8)$.

In this study, an in vivo quantification analysis, which evaluates the concentration of attached targeted MBs, is combined with ultrasound molecular imaging. We hypothesize that the concentration estimation (expressed as MBs per $\mathrm{mm}^{3}$ ) inside an ROI selected by observing the ultrasound image of the scene can support noninvasive tumor detection and evaluation. The method developed by us (9) for the estimation of the MB con-

\footnotetext{
* Correspondence to: Dr. Claudia Sciallero, Department of Electrical, Electronic, Telecommunications Engineering, and Naval Architecture (DITEN), University of Genoa, Via Opera Pia 11, 1-16145, Genoa, Italy.E-mail: claudia.sciallero@unige.it

a C. Sciallero, A. Trucco

Department of Electrical, Electronic, Telecommunications Engineering, and $\mathrm{Na}$ val Architecture (DITEN), University of Genoa, 16145, Genoa, Italy

b E. Daglio

Division of Urology, Ospedale Evangelico Internazionale, 16158, Genoa, Italy

c A. Trucco

Pattern Analysis \& Computer Vision, Istituto Italiano di Tecnologia (IIT), 16163, Genoa, Italy
} 
centration is significantly different from a straightforward analysis of the acoustic signal intensity. After a tissue rejection phase, i.e. a nonlinear imaging technique to abate the contribution from the surrounding tissue and to emphasize the MB echoes, a multi-dimensional vector of features is extracted from the residual backscattered signals. Subsequently, a nonlinear regression approach based on a support vector machine (SVM) is applied to estimate the MB concentration in small volumes (i.e. cubic voxels of $1 \mathrm{~mm}$ side lengths). To test our assumption, lipidic MBs containing targeting molecules were administered to rats bearing subcutaneous prostate tumors of different sizes. Targeted ultrasound imaging with a multi-pulse technique was performed with two different perfusion conditions to evaluate the contribution of targeted MBs. Radio-frequency (RF) signals were collected to quantify the MB concentration by off-line data processing. The obtained results were then correlated with the tumor extensions. To the best of our knowledge, this is the first study addressing the combination of molecular imaging and in vivo quantification analyses of targeted MBs.

\section{MATERIALS AND METHODS}

\subsection{Targeted CA}

In addition to the different vascular epithelial growth factor receptors (VEGFR), tissue factor (TF) is a potential vascular marker linked to tumor angiogenesis (10). TF has been reported to be expressed in tumor vessels in prostate cancer but not in the endothelium in healthy tissues (11). Soft-shell MBs (average diameter of $1.8 \mu \mathrm{m}$ ) with attached anti-TF antibodies were used as contrast media. The ligand density was on the order of $2 \times 10^{4}$ per MB. The average number of bound antibodies per square micrometer was approximately 2300 . In vitro and in vivo tests demonstrated the binding efficiency of these targeted MBs, and a significant signal enhancement was found with respect to non-targeted MBs $(12,13)$. Further details on the targeted CA used in this study can be found in $(12,13)$.

\subsection{Molecular imaging and $C A$ quantification}

Molecular imaging and CA quantification can be combined by processing the acquired RF data. A general approach was proposed by the authors in (9) and can be divided into three steps: 1) tissue rejections by means of a contrast-enhanced signal processing technique; 2 ) image generation and ROI localization; and 3) an estimation of the CA concentration.

In the first step, a non-destructive, multi-pulse imaging technique that avoids MB destruction by using moderate acoustic pressures was applied for CA detection. This technique is one of the contrast pulse sequences (CPS) based on the transmission of three pulses (14) and exploits the nonlinear backscattered signal generated by MBs. A given pulse scaled in amplitude by the coefficients $0.5,-1$, and 0.5 was transmitted three times, and the corresponding echoes were added with unitary coefficients. In this way, both the second- and third-order nonlinear terms are preserved, whereas the linear term, where tissues are predominant, is abated. A bandpass filter centered on the fundamental frequency can be conveniently applied to maintain only the third nonlinear component on the fundamental bandwidth, as discussed in (15). The processed RF signals are used to both generate the ultrasound image and to supply the input data for the concentration estimation procedure. In detail, a MATLAB (The MathWorks, Inc., MA, USA) script reads the RF frames that were acquired by the ultrasound scanner and organizes them into a three-dimensional (3D) matrix. The three dimensions of the matrix represent the number of lines of sight generated by the beamformer for the ultrasound image generation, the number of RF time samples for each line of sight, and the number of transmitted pulses (three for the CPS technique we adopted). The 3D matrix is reduced to a two-dimensional (2D) matrix by summing the three pulses that were acquired for each line of sight, according to the CPS strategy. Subsequently, as described above, band-pass filtering is applied through a FIR-filter that operates along the temporal dimension of the matrix. At this stage, the 2D RF data matrix can be used for ultrasound image rendering.

During the second step, the same functions as those typically used by the back-end of medical ultrasound scanners (i.e. envelope detection and logarithmic compression) were applied. After the image generation, an ROI was identified. The ROI identified a thin tissue slice whose surface is defined on the imaging plane and whose thickness (in the elevation plane, perpendicular to the imaging plane) can be considered constant. Thus, the ROI can be considered as a single layer of voxels whose minimum size depends on the resolution of the ultrasound imaging systems along the three orthogonal axes. Notably, because the 2D RF data matrix obtained at the end of the previous step corresponds to the imaging plane, each voxel contains $\Xi$ lines of sight and $\Omega$ time samples, depending on the voxel size.

In the last step, the concentration estimation was performed. First, the $\Xi$ RF signals and the $\Omega$ time samples contained inside each voxel of the ROI were identified on the 2D RF data matrix, depending on the specific position of the voxel. Then, for every RF signal segment contained inside a given voxel, the following features were computed: the signal energy, expressed on a logarithmic scale; the kurtosis of the normalized signal; the number of peaks of the normalized signal that exceed $20 \%$ of the signal maximum; the number of peaks of the signal envelope that exceed $90 \%$ of the envelope maximum; and the number of peaks of the signal spectrum whose magnitude is between the spectrum maximum minus $20 \mathrm{~dB}$ and the maximum itself. Thus, for each feature, $\Xi$ values were collected, i.e., one value for each line of sight contained inside the voxel. The final value of each feature of a given voxel was computed by averaging the $\Xi$ values.

To cope with a potential nonlinear dependency of the concentration from the extracted features, a nonlinear regression method based on the SVM was developed and experimentally validated (9) through in vitro tests, as briefly described in the next subsection. The training phase of the statistical regressor requires a large pool of signals generated from voxels that contain a given and well-known number of MBs. In addition, a large pool of signals is necessary for each possible concentration. The combination of these two requirements makes it extremely difficult (if not impossible) to collect all the signals necessary to build a satisfactory training set from real experiments. As a consequence, a numerical model that encompassed the transmission, propagation, tissue and $\mathrm{MB}$ scattering, reception and processing of the ultrasound signal was used to simulate the RF signal achieved for a given MB concentration $(9,15)$. The simulation model was generated according to the operating conditions used for the real experiments (e.g. probe characteristics, transmitted signal waveforms, imaging technique, focus depth, voxel size, and noise spectrum) and was calibrated through the analysis of some real signals (9), as described in the next subsection. Thus, a completely synthetic training phase was possible. The regression model for the relationship between the features and 
MB concentration obtained from the training phase was then applied to estimate the concentration, taking as input the features extracted from real signals.

\subsection{In vitro calibration and validation}

In vitro experiments were performed under controlled conditions (9) to calibrate the simulation model and test the accuracy of the concentration estimator based on a synthetic training phase.

As reported in (9), an inverted brightfield/phase contrast microscope equipped with a fast greyscale camera was used to count the number of bubbles (i.e. the real concentration) attached to a wall of a small flow channel immersed in a material that mimics tissue and is optically and acoustically transparent. The microscopy images represent $1 \mathrm{~mm}^{3}$ of the upper wall of the channel. Different simultaneous optical-acoustic acquisitions were performed to compare real and estimated concentrations according to the setup conditions reported in (9). During these tests, seven concentrations were observed using the optical equipment: $11,19,36,54,75,86$, and $98 \mathrm{MBs} / \mathrm{mm}^{3}$. For each concentration, the ultrasound backscattered signals were acquired several consecutive times.

Such signals were used to derive the average contrast to tissue ratio (CTR, i.e. the ratio between the received energy of the $M B$ response and that of the tissue response) as a function of $M B$ concentration. The observed CTR curve was used to weight the tissue and $M B$ responses generated by the simulation model before their final addition in such a way that simulated and backscattered signals showed similar CTR values. In this way, a calibrated simulation model was attained.

Then, our concentration estimation method based on a synthetic training phase (performed using signals that were generated by the calibrated simulation model) was validated by comparing the estimated and real concentration values. The results of this test are described in (9); in general, the absolute value of the average error did not exceed $4 \mathrm{MBs} / \mathrm{mm}^{3}$. An error of $5 \mathrm{MBs} / \mathrm{mm}^{3}$ was reported for only one concentration. Although these results represented positive experimental validation of the proposed method, this previous study failed to evaluate the achievable performance when the training phase is performed using real signals (9). We used the same signals and methods as in (9) to perform this evaluation; we compared the estimations obtained when the training set was composed of synthetic signals with those obtained when the training set was composed of real signals. The test set was the same for the two training options and was composed of real signals. Regardless, the signals for the test set were different from those used for the training phase. The comparison of the results obtained with the two training options demonstrated that for all the $\mathrm{MB}$ concentrations considered in this paper, the differences between the average estimated values did not exceed 4 $\mathrm{MBs} / \mathrm{mm}^{3}$. Therefore, we verified that the concentration estimations obtained by training the statistical regressor with synthetic signals (generated using the calibrated simulation model) were close to the real values, and furthermore, they were close to the values estimated using real signals for the training phase.

\subsection{In vivo experiments}

Targeted MBs were injected intravenously via the tail vein of adult rats bearing subcutaneous VCaP prostate cancers. For each injection, $400 \mu \mathrm{L}$ of $\mathrm{CA}\left(10^{8} \mathrm{MBs} / \mathrm{ml}\right)$ was administered and flushed with $600 \mu \mathrm{L}$ of sterile saline. Approximately $4 \times 10^{7}$ $\mathrm{MBs}$ were injected into a blood volume of approximately $50 \mathrm{~mL}$ to obtain a concentration of $8 \times 10^{5} \mathrm{MBs} / \mathrm{mL}$ (equivalent to $8 \times 10^{7} \mathrm{MBs} / \mathrm{kg}$ ), which is on the order of the concentration used for a human bolus injection (16). A modified version of a MyLab70XVG ultrasound scanner (Esaote S.p.A., Genoa, Italy) developed for research purposes and equipped with a special frame grabber board that collects the RF signals at the beamformer output was used. The ultrasound scanner was connected to a linear array probe with a central frequency of $5 \mathrm{MHz}$ and a fractional bandwidth of $90 \%$. To avoid any movement concerns and related artefacts, the ultrasound probe was mechanically fixed using an articulated arm, and the animals were anesthetized. The pulses transmitted according to the CPS technique were 5-cycle, Gaussian-shaped signals with a central frequency of $5.2 \mathrm{MHz}$. For reception, the grabber board collected the three backscattered signals for each line of sight. For all experiments, the negative peak pressure at the focus depth (placed $1.7 \mathrm{~cm}$ below the probe) was $100 \mathrm{kPa}$, corresponding to a mechanical index (MI) of 0.04. Due to the specific characteristics of the adopted probe and pulses, the axial, lateral and elevation resolutions enabled the definition of cubic voxels with $1 \mathrm{~mm}$ sides. The numbers of lines of sight and time samples of the 2D RF data matrix contained inside each voxel were $\Xi=6$ and $\Omega=80$, respectively. In Fig. 1, the mean acoustic intensity in the ROI (i.e. a well-perfused area inside the tumor and surrounding neovessels) was monitored for $6 \mathrm{~min}$. RF signals were acquired every $30 \mathrm{~s}$ and were processed using a script. A significant contrast-enhancement due to flowing and adherent MBs was recorded following $C A$ injection. A plateau was reached after $3.5 \mathrm{~min}$. To evaluate the concentration of adherent MBs, the image was analyzed under two perfusion conditions. A first RF signal acquisition was performed between 3.5 and 4 min after the CA injection. The acquired signals contained the contribution of flowing and adherent MBs. Immediately after, the MBs were destroyed by a high-intensity pulse (an acoustic pressure higher than $1 \mathrm{MPa}$, corresponding to an $\mathrm{Ml}$ of 0.5 ). Thirty seconds after the destruction, RF signals were acquired for a second time. In this case, the signals mainly contained contributions from the flowing MBs because in a $30 \mathrm{~s}$ interval, the MBs could not adhere to target sites (12). A similar protocol was used for the ultrasound image analysis of targeted MBs in (5). The concentration of attached MBs was estimated starting from the two data acquisition periods: the final estimate was derived

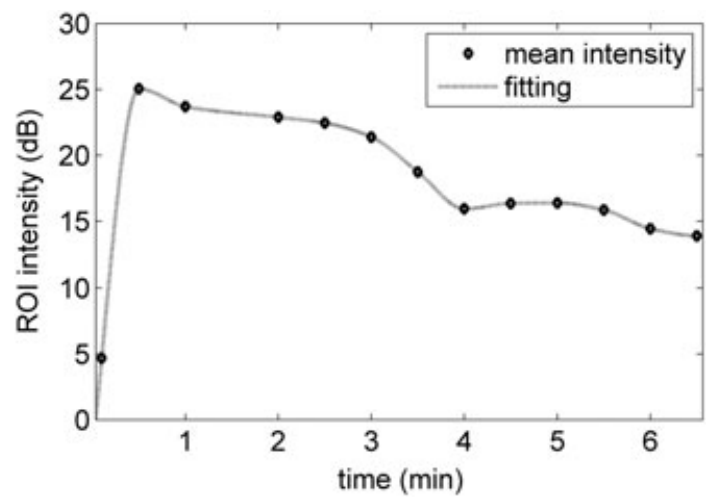

Figure 1. Average acoustic intensity in the ROI (well-perfused areas in the tumor and surrounding vessels) for $6 \mathrm{~min}$ after the injection of targeted MBs and the intensity curve trend using a $6^{\text {th }}$ order polynomial fit. 
by subtracting the concentration estimated after the $M B$ destruction from the concentration estimated before the $M B$ destruction.

\section{RESULTS AND DISCUSSION}

Initially, the subcutaneous tumors were imaged in B-mode (i.e. on-line image acquisition in standard mode) without the administration of CA. Because all tumors were confined to a specific area (i.e. the encapsulated tumors), the sizes were recorded by means of the electronic calipers on the ultrasound system. An example is shown in Fig. 2. The four animals were analyzed, and the areas of their tumors on the ultrasound images were $27,33,54$, and $94 \mathrm{~mm}^{2}$. In the first two cases, the sizes were similar.

After the CA administration, the previously described protocol was applied. In Fig. 3, the contrast-enhanced ultrasound images obtained from the off-line processing of the signals acquired before and after the MB destruction are shown. As seen in Fig. 3(a), acquired $4 \mathrm{~min}$ after administration, the flowing and adherent MBs were present. As seen in Fig. 3(b), acquired 4.5 min after administration (which corresponded to $30 \mathrm{~s}$ after the MB destruction), flowing MBs were primarily present. To emphasize the contribution of the adherent MBs, the difference image was computed. This result is shown in Fig. 3(c) in a logarithmic scale (as for the previous images) and a false-color palette. A few spurious artefact contributions and speckle noise are present but are easily distinguishable.

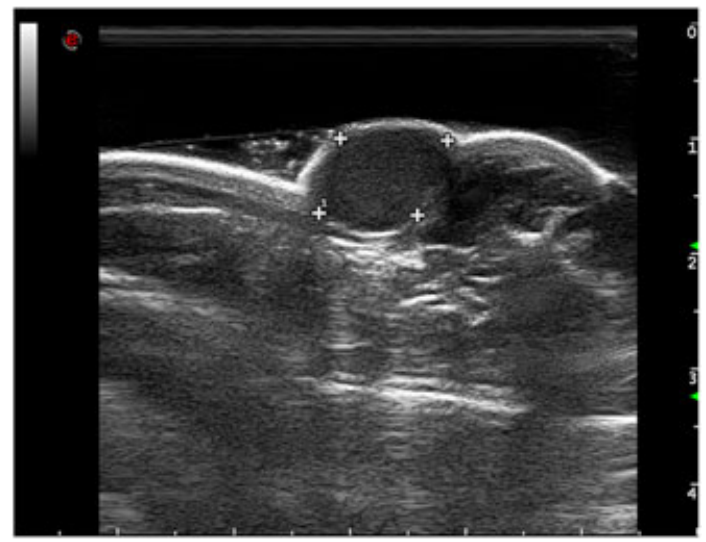

Figure 2. B-mode ultrasound image of the subcutaneous tumor acquired during the size evaluation phase. CA was not administered.
The visual analysis of these images enabled the delineation of a rectangular $\mathrm{ROI}$ for each examined tumor. The ROls were regions inside which the estimations of the $M B$ concentrations were performed. The ROls were placed around the focus depth $(1.7 \mathrm{~cm}$ below the probe, close to the lower interface between the tumor and tissues) and included well-perfused areas inside the tumor and surrounding neovascular tissues. In Fig. 4, the contrast-enhanced ultrasound images of the four tumors acquired before and after the MB disruption and the related concentration maps are shown. The position of the ROI for each tumor is specified by the superposition of a red grid on the corresponding ultrasound images. A concentration map shows the estimated concentration of adherent MBs for each cubic voxel in the related ROI. The voxels composing an $\mathrm{ROI}$ are represented in Fig. 4 as a grid of small squares whose grey levels express the estimated concentrations.

To support the analysis of the results and to compare the four experiments, Fig. 5 displays two histograms that show the following for each tumor: (a) the sample mean and standard deviation of the estimated MB concentration inside the related ROI; and (b) the relative frequency of the voxels with estimated $M B$ concentrations of less than $10 \mathrm{MBs} / \mathrm{mm}^{3}$, greater than 20 $\mathrm{MBs} / \mathrm{mm}^{3}$, and greater than $30 \mathrm{MBs} / \mathrm{mm}^{3}$. The correlation between the tumor size and the estimated MB concentration was determined using a correlation coefficient $r$, keeping the $p$ value less than 0.05 . We verified the existence of a significant correlation between the tumor size and the sample mean of the estimated attached MBs concentration in the ROI ( $r=0.877$, $p<0.05$ ). Moreover, the analysis of the second graph reveals that concentrations less than $10 \mathrm{MBs} / \mathrm{mm}^{3}$ are predominant when small tumors are considered $(r=0.793, p<0.05)$, whereas the percentage of voxels with a concentration greater than 20 $\mathrm{MBs} / \mathrm{mm}^{3}$ increases with tumor size $(r=0.893, p<0.05)$. Finally, for the tumors with sizes of $27 \mathrm{~mm}^{2}$ and $33 \mathrm{~mm}^{2}$, the estimated concentrations for the ROI voxels did not exceed $30 \mathrm{MBs} / \mathrm{mm}^{3}$, whereas for the $94 \mathrm{~mm}^{2}$ tumor, the percentage of voxels with concentrations greater than $30 \mathrm{MBs} / \mathrm{mm}^{3}$ was approximately $50 \%$. The correlation observed between the relative frequency of voxels that exceeded $30 \mathrm{MBs} / \mathrm{mm}^{3}$ and the tumor size was particularly high $(r=0.944, p<0.05)$.

The correlation between the tumor size and the estimated CA concentration can be explained as follows. The growth of most solid tumors is highly dependent on local vascular proliferation (4). A higher vascularization with an increased cancer cell density and molecular markers occurs when the size of the tumor increases. Consequently, the increased number of target sites facilitates the accumulation of targeted MBs. The proposed

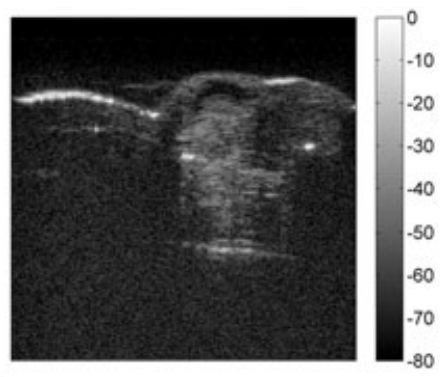

(a)

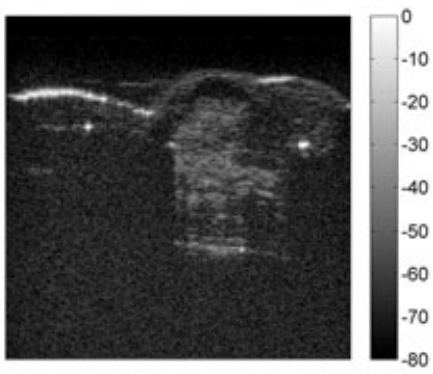

(b)

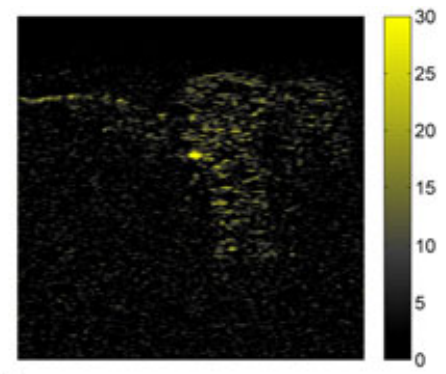

(c)

Figure 3. Contrast-enhanced ultrasound images acquired: (a) 4 min after CA injection; (b) 30 s after MB destruction (i.e., approximately 4.5 min after CA injection). The difference image (c) shows the attached MB contribution. The image brightness is expressed on a logarithmic scale. 
(a)

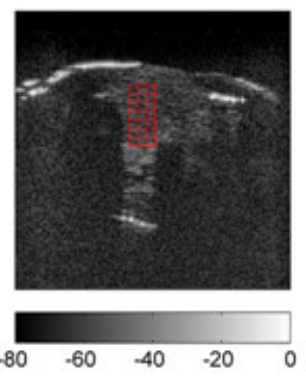

(b)

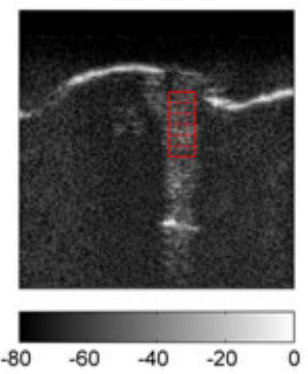

(c)

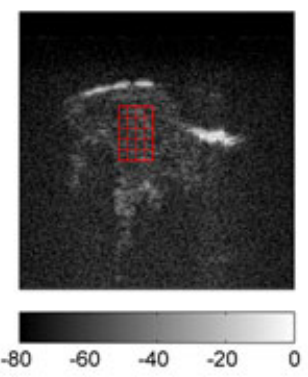

(d)

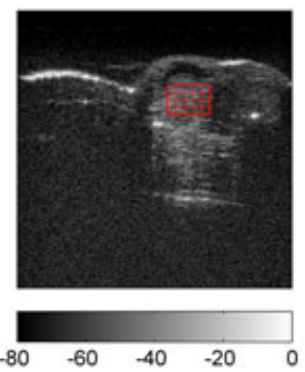

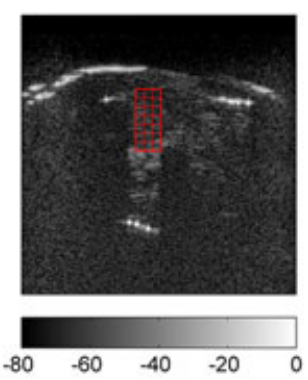
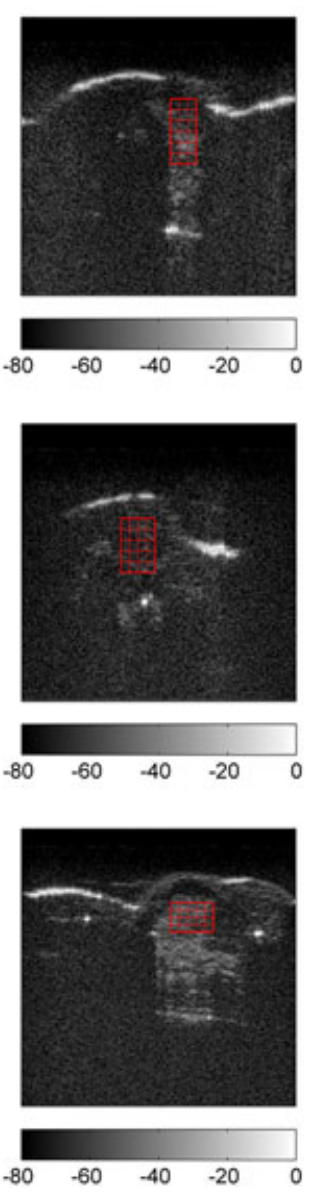
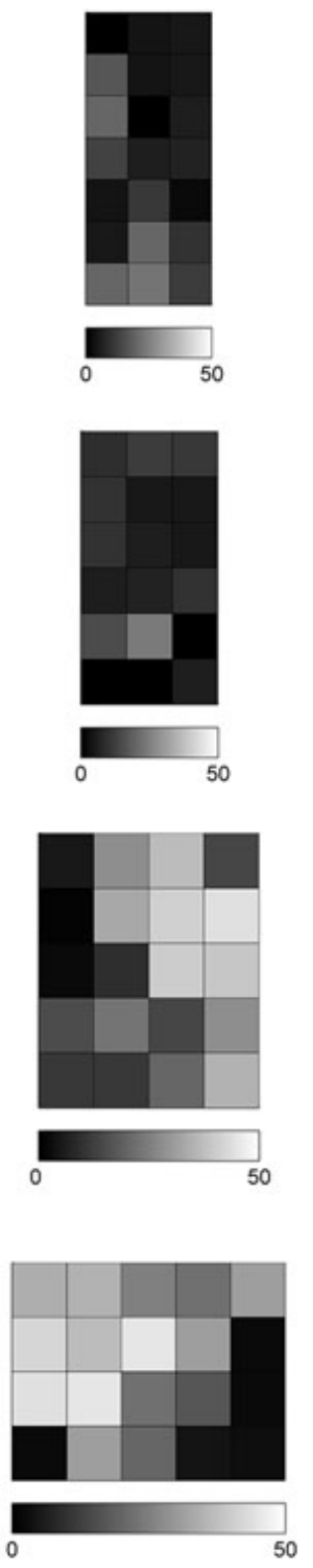

Figure 4. Ultrasound images before (left column) and after MB disruption (center column) and the related concentration maps (right column) for the adherent targeted MBs. The tumor sizes for the four analyzed mice were: (a) $27 \mathrm{~mm}^{2}$; (b) $33 \mathrm{~mm}^{2}$; (c) $54 \mathrm{~mm}^{2}$; and (d) $94 \mathrm{~mm}^{2}$. The image brightness is on a logarithmic scale; the CA concentration is on a linear scale, measured in $\mathrm{MBs} / \mathrm{mm}^{3}$.

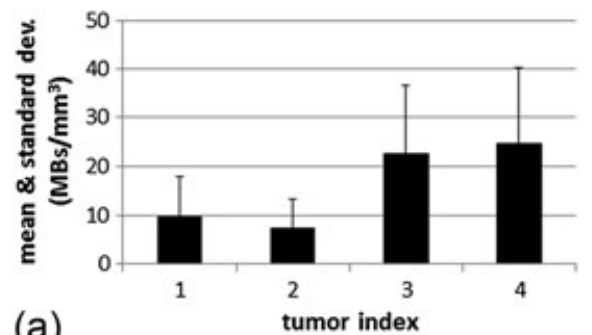

(a)

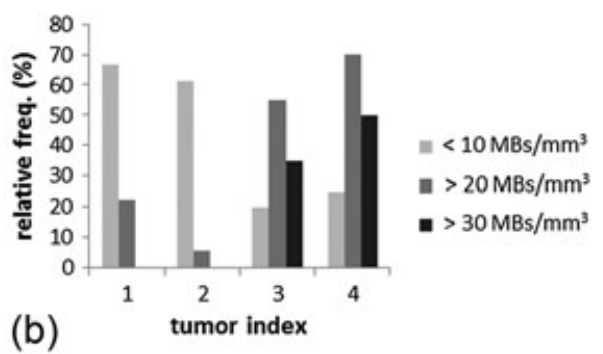

Figure 5. (a) Sample mean and standard deviation of the MB concentration estimated for each tumor. (b) Relative frequency of the voxels with an estimated MB concentration less than $10 \mathrm{MBs} / \mathrm{mm}^{3}$, greater than $20 \mathrm{MBs} / \mathrm{mm} 3$ and greater than $30 \mathrm{MBs} / \mathrm{mm}^{3}$. The four experiments are indexed from 1 to 4 according to the tumor size, in ascending order.

quantification method, based on a nonlinear regression technique, is a powerful tool that is more sensitive and accurate than conventional image intensity analysis methods (9).
Considering that the subtraction procedure used in this study is prone to motion artefacts, it is interesting to note that the freeflowing MB contribution can be rejected by strategies that do 
not require a $M B$ destruction step and that are more compliant with clinical practice (even if they can be less accurate in rejecting the flowing $\mathrm{MB}$ contribution). For instance, in (7), the free-flowing MBs are distinguished from attached MBs by waiting for a sufficiently long time interval (e.g. seven minutes from the MB injection) to allow both $\mathrm{MB}$ accumulation at target sites and unbound MB clearance. This alternative protocol can be easily adopted in combination with the concentration estimation method that we proposed to correlate MB accumulation with tumor size.

\section{CONCLUSIONS}

In vivo experiments were performed to explore the possibility of combining molecular imaging with targeted CA quantification to obtain additional information useful for cancer assessment. The results demonstrated a significant correlation between the size of the tumor and the estimated concentrations of the adherent MBs. Thus, CA quantification has the potential to support the early diagnosis of cancer pathology by localizing and evaluating tiny quantities of neoplastic tissues that are difficult to assess by conventional ultrasound image intensity analyses. Moreover, the combination of molecular imaging and CA quantification represents additional degrees of assessment after basic diagnostic procedures (e.g. PSA and DRE screening in the case of prostate cancer). The improvement of the cancer detection rate during biopsy exams is another potential medical application. An echoguided biopsy supported by the quantification of the targeted CAs in the ROI could help prevent a high number of false negatives and enhance the exam sensitivity.

\section{Acknowledgements}

The authors are grateful to Prof. F. Frauscher, Dr. J. Gradl and Dr. M. Abdellah for animal preparation and their assistance with the experiments. Additionally, the assistance of Dr. P. Pellegretti and Dr. M. Crocco with the data acquisition is gratefully acknowledged. The authors also thank Esaote S.p.A., Genova, Italy, for the equipment support.

\section{REFERENCES}

1. Klibanov AL. Ultrasound molecular imaging with targeted microbubble contrast agents. J Nucl Cardiol 2007; 14: 876-884.
2. Cosgrove D. Ultrasound contrast agents: an overview. Eur Radiol 2006; 60: 324-330.

3. Hauff $P$, Reinhardt M, Foster S. Ultrasound contrast agents for molecular imaging. In Handbook of Experimental Pharmacology. Molecular Imaging I, Semmer W, Schwaiger M (eds). Springer-Verlag: Berlin, 2008; 223-245.

4. Ellegala DB, Leong-Poi H, Carpenter JE, Klibanov AL, Kaul S, Shaffrey $\mathrm{ME}$, Sklenar J, Lindner JR. Imaging tumor angiogenesis with contrast ultrasound and microbulles targeted to $\alpha_{v} \beta_{3}$. Circulation 2003; 108: 336-341

5. Stieger SM, Dayton PA, Borden MA, Caskey CF, Griffey SM, Wisner ER, Ferrara KW. Imaging of angiogenesis using Cadence ${ }^{T M}$ contrast pulse sequencing and targeted contrast agents. Contrast Media Mol Imaging 2008; 3: 9-18.

6. Deshpande N, Needles A, Willmann JK. Molecular ultrasound imaging: current status and future directions. Clin Radiol 2010; 65: 567-581.

7. Anderson CR, Hu X, Tlaxca J, Decleves A-E, Houghtaling R, Sharma K, Lawrence M, Ferrara K, Rychak JJ. Ultrasound molecular imaging of tumor angiogenesis with an integrin targeted microbubble contrast agent. Invest Radiol 2011; 46(4): 215-224.

8. Halpern EJ, Rosenberg M, Gomella LG. Prostate cancer: contrastenhanced US for detection. Radiology 2001; 219: 219-225.

9. Sciallero C, Crocco M, Trucco A. A method for estimating the microbubble concentration in contrast-enhanced ultrasound imaging. Meas Sci Technol 2011; 22: 114009 (9pp).

10. Takano S, Tsuboi K, Tomono Y, Mitsui Y, Nose T. Tissue factor, osteopontin, $\alpha_{v} \beta_{3}$ integrin expression inmicrovasculature of gliomas associated with vascular endothelial growth factor expression. $\mathrm{Br} J$ Cancer 2000; 82(12): 1967-1973.

11. Hu Z, Garen A. Targeting tissue factor on tumor vascular endothelial cells and tumor cells for immunotheraphy in mouse models of prostatic cancer. Proc Natl Acad Sci U S A 2001; 98(21): 12180-12185.

12. Zerbe I, Gajovic-Eichelmann N. Lipidic microbubble targeting of surface proteins using an in vitro system. In Ultrasound contrast agents. Targeting and processing methods for theranostics, Paradossi G, Pellegretti P, Trucco A (eds). Springer-Verlag: Milan, 2010; 41-52.

13. Hauwel $M$, Bettinger $T$, Allemann E. Use of microbubbles as ultrasound contrast agents for molecular imaging. In Ultrasound contrast agents. Targeting and processing methods for theranostics, Paradossi G, Pellegretti P, Trucco A (eds). Springer-Verlag: Milan, 2010; 13-23.

14. Phillips PJ. Contrast pulse sequences (CPS): imaging nonlinear microbubbles. Proc. 2001 IEEE Ultrasonics Symposium, Atlanta, GA, 2001; 2: 1739-1745.

15. Crocco M, Palmese M, Sciallero C, Trucco A. A comparative analysis of multi-pulse techniques in contrast-enhanced ultrasound medical imaging. Ultrasonics 2009; 49: 120-125.

16. Schneider M. Characteristics of SonoVue ${ }^{\mathrm{TM}}$. Echocardiography 1999; 9(7): 743-746. 